\title{
Serum MicroRNA Signature as a Diagnostic and Therapeutic Marker in Patients with Psoriatic Arthritis
}

\author{
Sarah M. Wade ${ }^{1} \mathbb{D}$, Trudy McGarry ${ }^{1}$, Siobhan C. Wade ${ }^{2}$, Ursula Fearon ${ }^{1}$, and Douglas J. Veale
}

\begin{abstract}
Objective. MicroRNA (miRNA) are small endogenous regulatory RNA molecules that have emerged as potential therapeutic targets and biomarkers in autoimmunity. Here, we investigated serum miRNA levels in patients with psoriatic arthritis (PsA) and further assessed a serum miRNA signature in therapeutic responder versus nonresponder PsA patients.

Methods. Serum samples were collected from healthy controls (HC; $\mathrm{n}=20)$ and PsA patients $(\mathrm{n}=31)$, and clinical demographics were obtained. To examine circulatory miRNA in serum from HC and PsA patients, a focused immunology miRNA panel was analyzed utilizing a miRNA Fireplex assay (FirePlex Bioworks Inc.). MiRNA expression was further assessed in responders versus nonresponders according to the European League Against Rheumatism response criteria.

Results. Six miRNA (miR-221-3p, miR-130a-3p, miR-146a-5p, miR-151-5p, miR-26a-5p, and miR-21-5p) were significantly higher in PsA compared to HC (all $P<0.05$ ), with high specificity and sensitivity determined by receiver-operating characteristic curve analysis. Analysis of responder versus nonresponders demonstrated higher baseline levels of miR-221-3p, miR-130a-3p, miR-146a-5p, miR-151-5p, and miR-26a-5p were associated with therapeutic response.

Conclusion. This study identified a 6-serum microRNA signature that could be attractive candidates as noninvasive markers for PsA and may help to elucidate the disease pathogenesis.
\end{abstract}

Key Indexing Terms: autoimmune diseases, inflammation, psoriatic arthritis

Small noncoding RNA called microRNA (miRNA) that function as posttranscriptional regulators of target genes have become increasingly recognized as key regulators in the development of autoimmune disease ${ }^{1}$. In addition to being capable of modulating numerous autoimmunity-related genes ${ }^{2,3,4}$, several studies have also reported the potential utilization of circulating miRNA [serum, plasma, and peripheral blood mononuclear cells $(\mathrm{PBMC})]$ as predicative noninvasive biomarkers of therapeutic response $e^{5,6}$.

As a complex, chronic, multifaceted, and progressive autoinflammatory disease, psoriatic arthritis (PsA) would greatly benefit from markers of early diagnosis and therapeutic intervention to effectively suppress inflammation and control disease

The authors would like to acknowledge the financial support from the Maeve Binchy Funding for Arthritis Research and the IRC postgraduate fellowship.

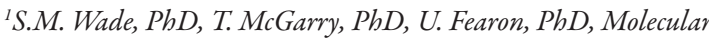
Rheumatology, School of Medicine, Trinity Biomedical Sciences Institute, Trinity College Dublin, and Centre for Arthritis and Rheumatic Disease, St. Vincent's University Hospital, and University College Dublin; ${ }^{2}$ S.C. Wade, $\mathrm{PhD}$, Molecular Rheumatology, School of Medicine, Trinity Biomedical Sciences Institute, Trinity College Dublin; ${ }^{3} D$.J. Veale, MD, Centre for Arthritis and Rheumatic Disease, St. Vincent's University Hospital, and University College Dublin, Dublin, Ireland.

Address correspondence to Prof. D.J. Veale, Centre for Arthritis and Rheumatic Disease, St. Vincent's University Hospital, and University College Dublin, Dublin 4, Ireland.Email: douglas.veale@ucd.ie.

Full Release Article. For details see Reprints and Permissions at jrheum.org. Accepted for publication February 13, 2020. progression $^{7,8}$. The multifaceted presentation of synovial inflammation, joint destruction, enthesitis, axial disease, and skin manifestations often makes diagnosis difficult, with as many as $15-21 \%$ of patients remaining undiagnosed in dermatology clinics 9 . Diagnostic delays as short as 6 months might result in elevated bone erosion rates and significant deterioration in long-term physical function ${ }^{10}$. While the treatment of PsA has greatly improved in the last decade ${ }^{11}$, up to $47 \%$ of patients show evidence of bone erosions within the first 2 years of initial diagnosis, despite the use of conventional disease-modifying agents ${ }^{12}$.

MiRNA have been shown to be altered in the synovial tissue and PBMC of patients with PsA ${ }^{13,14}$, supporting miRNA as possible diagnostic and prognostic biomarkers for PsA. However, no studies have evaluated serum miRNA profiles in early PsA patients compared to healthy controls (HC) or assessed the ability of baseline miRNA expression to stratify patient outcomes to treatment. Therefore, the present study was designed to evaluate the expression profile of circulating miRNA, specifically focusing on a defined immunology miRNA panel, in patients with PsA to identify miRNA associated with the disease and with response to therapy.

\section{MATERIALS AND METHODS}

Patient recruitment. Thirty-one patients with PsA were recruited from the outpatient clinics at the Centre for Arthritis and Rheumatic Diseases, St Vincent's University Hospital, Dublin. All patients fulfilled the ClASsification for Psoriatic ARthritis criteria (CASPAR) criteria for the classification of $\mathrm{PsA}^{15}$. All patients were naïve to treatment and had active inflammation in one or both knees at the time of assessment. The control

Personal non-commercial use only. The Journal of Rheumatology Copyright @ 2020 . All rights reserved. 
group consisted of 20 healthy individuals who were negative for autoimmune diseases.

Baseline clinical and laboratory assessments included C-reactive protein (CRP), erythrocytes sedimentation rates (ESR), swollen joint count (SJC), and tender joint count (TJC).

A group of 31 patients with follow-up clinical data were classified as good, moderate, and nonresponders to treatment according to the European League Against Rheumatism (EULAR) response criteria as previously described ${ }^{16,17,18,19}$. Assessments were made independent of treatment type [disease-modifying antirheumatic drugs (DMARD)] or time (3 mos, $6 \mathrm{mos}$, and $9 \mathrm{mos}$ ). Macroscopic synovitis and vascularity were quantified under direct visualization at arthroscopy using a well-established visual analog scale (VAS) $1-100 \mathrm{~mm}$ as previously described ${ }^{20}$.

All research was performed in accordance with the Declaration of Helsinki, and approval for this study was granted by the St. Vincent's University Hospital Medical Research and Ethics Committee (RHEV7_09/16). All patients gave fully informed written consent prior to inclusion.

FirePlex serum miRNA expression analysis. Peripheral blood was collected in anticoagulant-free tubes for obtaining serum. All bloods were processed within $1 \mathrm{~h}$ of collection. Samples were centrifuged at $2000 \mathrm{~g}$ for $10 \mathrm{~min}$ at room temperature. Isolated serums were transferred to RNAse- and DNAse-free tubes and stored at $-80^{\circ} \mathrm{C}$ until required for further analysis. Rather than performing an exploratory study examining $100 \mathrm{~s}$ of miRNA, we performed a focused immunology panel, designed based on a detailed analysis of published miRNA data by the Abcam Fireplex miRNA Biofluid assay (FirePlex Bioworks Inc.), as previously described ${ }^{21}$. Briefly, a focused panel of 68 miRNA were analyzed in $20 \mu \mathrm{L}$ of serum by the Abcam Fireplex miRNA Biofluid assay. Target miRNA were hybridized to miRNA target sequences on hydrogel FirePlex particles $(35 \mu \mathrm{L})$ at picomolar sensitivity and high specificity following incubation at $37^{\circ} \mathrm{C}$ for $60 \mathrm{~min}$ with shaking. Bound miRNA were labeled with fluorescent adaptor molecules $(75 \mu \mathrm{L}$ of Labelling Buffer) and incubated at room temperature for $60 \mathrm{~min}$ with shaking. Eluted miRNA/adaptor complexes were amplified using single-step universal primer RT amplification consisting of 27 cycles of PCR amplification followed by 6 cycles of asymmetric amplification. Amplified products were rehybridized and incubated with $75 \mu \mathrm{L}$ of Reporting Buffer at room temperature for $15 \mathrm{~min}$ with shaking. Following the addition of $175 \mu \mathrm{L}$ of Run Buffer, miRNA particles were quantitatively detected on an EMD Millipore Guava 8HT flow cytometer and analyzed by the FirePlex Analysis Workbench (FirePlex Bioworks). Red blood cell contamination was assessed by miRNA hemolysis makers: hsa-miR-451a and hsa-miR-486-5p $\mathrm{p}^{22}$. Samples were also corrected for background signals determined by positive, negative, and blank control wells. The GeNorm normalization strategy was used for normalization ${ }^{23}$. Heatmaps were generated in FirePlex Analysis Workbench and hierarchical clustering was performed with weighted and Ward linkage. Six statistically significant miRNA (Bonferroni-corrected, $P \leq 0.02$ ) were chosen for further analysis.

Statistical analysis. The statistical significance of intergroup differences was determined by Kruskal-Wallis 1-way ANOVA test comparing the median values among all groups. Datasets for pair-wise analysis were assessed using the D'Agostino-Pearson, and/or Shapiro-Wilk normality test to confirm Gaussian distribution, followed by $t$ tests to determine statistical significance. Unsupervised 2-way hierarchical clustering algorithm based on Ward linkage and weighted linkage correlation metric was performed based on the normalized expression profiles from the top 20 of the most variable miRNA across all samples. The predicted probability of being a good, moderate, or nonresponder was used to construct receiver-operating characteristic (ROC) curves. Area under the ROC curve (AUC) with 95\% CI, sensitivity, specificity, and likelihood ratios were calculated to determine the accuracy index for evaluating the predictive performance of the selected miRNA. Spearman rank correlation coefficient was used to evaluate the association between miRNA levels and clinical variables.

\section{RESULTS}

Distinct miRNA expression profile in PsA patient serum compared to $H C$. To evaluate the relationship of serum miRNA expression profiles in early PsA patients and HC, we profiled miRNA signatures in 31 early PsA patients naïve to treatment and 20 HC subjects (Figure 1). The baseline characteristics of the patients are provided in Supplementary Table 1 (available with the online version of this article). Using the high throughput Firefly Bioworks miRNA-profiling technology, we focused on a 68-miRNA immunology panel based on their association with immune dysfunction (Supplementary Table 2). Unsupervised hierarchical clustering of the 20 most differentially expressed miRNA between PsA and HC serum showed independent clustering of PsA patient and HC groups (Figure 2), suggesting distinct miRNA serum profiles in PsA patients compared to $\mathrm{HC}$, with only 3 of the 31 PsA patients incorrectly clustered within the HC cluster. In this profile, 14 miRNA showed elevated expression, while 6 miRNA were decreased compared to HC (Supplementary Table 3). Among the identified miRNA, 6 miRNA were significantly overexpressed in PsA compared to $\mathrm{HC}$ and were further analyzed: miR-221-3p, miR-130a-3p, miR-146a-5p, miR-151-5p, miR-26a-5p (all $P<0.001$ ) and miR-21-5p $(P<0.01$; Figure 3$)$. ROC analysis was performed to determine the sensitivity and specificity of these miRNA to discriminate between PsA and HC (Figure 4). Levels of miR-130a-3p and miR-26a-5p showed strong statistical separation between PsA and controls with an AUC of 0.863 and 0.892 , respectively. Multivariate analysis for the strongest performing miRNA and the complete 6 miRNA signature did not improve the predictive value as compared to the individual miRNA (Supplementary Figure 1). Further, to ensure that the differential expression of miR-221-3p, miR-130a-3p, miR-146a-5p, miR-151-5p, miR-26a-5p, and miR-21-5p observed in PsA serum is owing to disease-specific associations, miRNA serum expression was compared to the nonspecific markers of inflammation, ESR, and CRP (Supplementary Figures 2 and 3). Among the 6 differentially expressed miRNA, only miR-130a-3p and miR-146a-5p expression levels correlated with CRP levels $(P=0.004, r=0.52$ and $P=0.03, r=0.41$, respectively), with no significant correlation observed between these differentially expressed miRNA and the ESR.

Baseline serum miRNA expression predict treatment response. Treatment response was determined according to the EULAR response criteria as previously described ${ }^{16,17,18,19}$, independent of type or duration of medication. Baseline characteristics of the study population separated according to their follow-up treatment response status are presented in Supplementary Table 4 (available with the online version of this article). We found that demographic variables (age, sex), clinical scores (SJC, TJC, VAS), and markers of synovial inflammation (synovitis, vascularity) were not significantly different across good/moderate and nonresponder PsA patients at baseline. In contrast to this, we demonstrated that baseline serum expression of miR-130a-3p $(P<0.01)$, miR-221-3p, miR-146a-5p, miR-151a-5p, and

Personal non-commercial use only. The Journal of Rheumatology Copyright $\subset$ 2020. All rights reserved. 


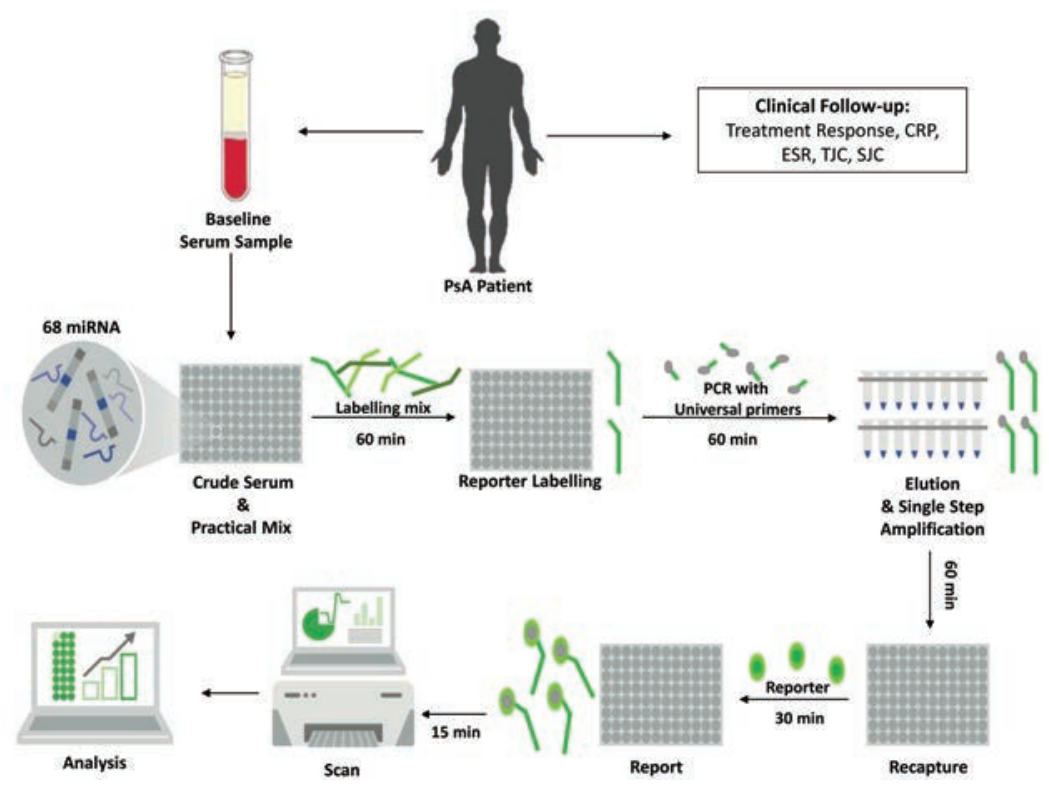

Figure 1. Process of PsA patient serum miRNA analysis. PsA patients naïve to treatment were recruited at baseline and their clinical progression followed. Fireplex Biofluid miRNA assays (FirePlex Bioworks Inc.) with 68 immune-associated miRNA were used to identify candidate diagnostic biomarkers in a quick and easy step-wise protocol. Baseline miRNA levels were retrospectively stratified to identify potential serum miRNA biomarkers of treatment response in early disease. CRP: C-reactive protein; DAS28: Disease Activity Score in 28 joints; ESR: erythrocyte sedimentation rate; miRNA: microRNA; PsA: psoriatic arthritis; SJC: swollen joint count; TJC: tender joint count. Image has been modified with permission from the FirePlex miRNA assay technical note. Abcam, Inc. hereby authorizes The Journal of Rheumatology Co. Ltd to publish the attached image [Figure 1 in Fearon publication] in The Journal of Rheumatology. This image may be modified by The Journal of Rheumatology Co. Ltd and used for subsequent publication upon written mutual agreement between Abcam, Inc. and The Journal of Rheumatology Co. Ltd.

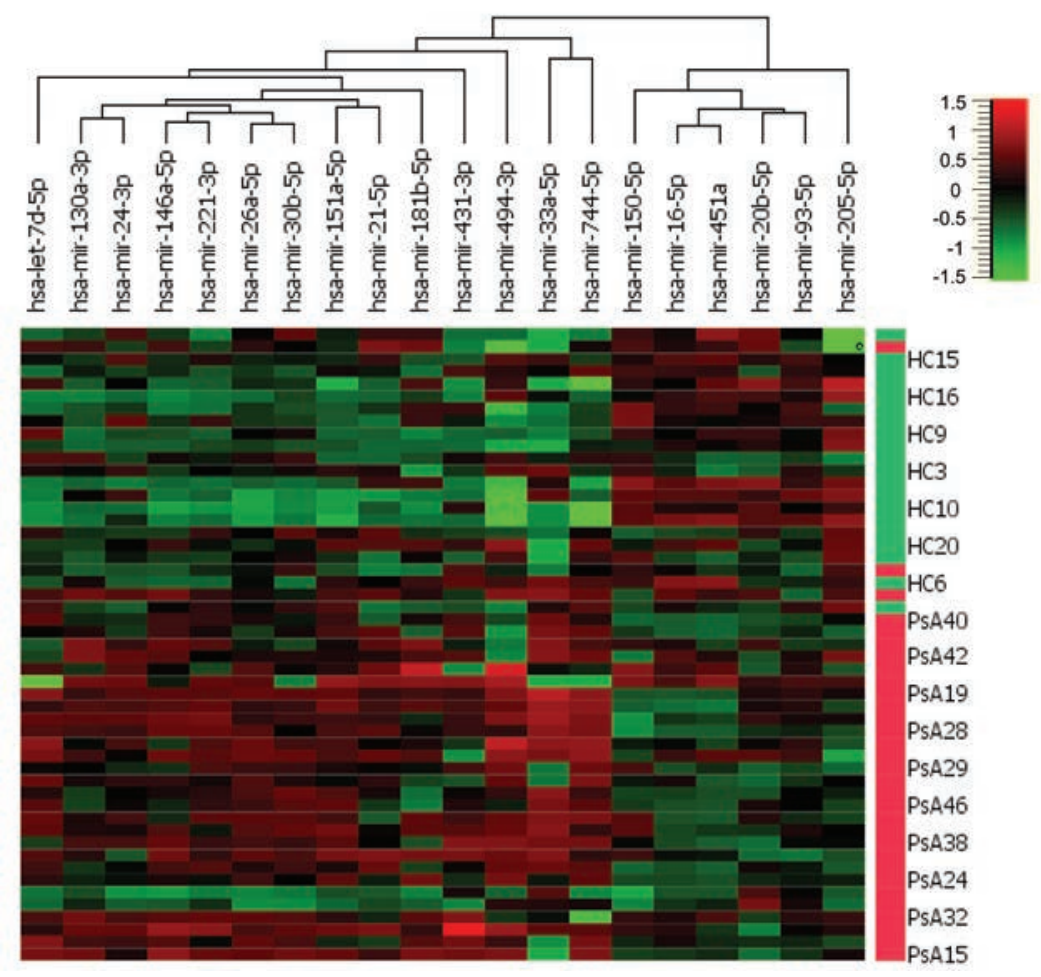

Figure 2. Hierarchical clustering analysis of miRNA expression for the top 20 differentially expressed miRNA in early PsA. Heatmap of individual serum miRNA expression in 31 patients with PsA and 20 HC. Each column represents single miRNA and each row represents individual samples. The color key indicates the degree of differential expression: green $=$ lower expression; black = no change, and red $=$ higher expression. Two major clusters were identified by unsupervised 2-way hierarchical miRNA (weighted linkage) and sample (Ward linkage) clustering analysis. HC: healthy controls; miRNA: microRNA; PsA: psoriatic arthritis. 

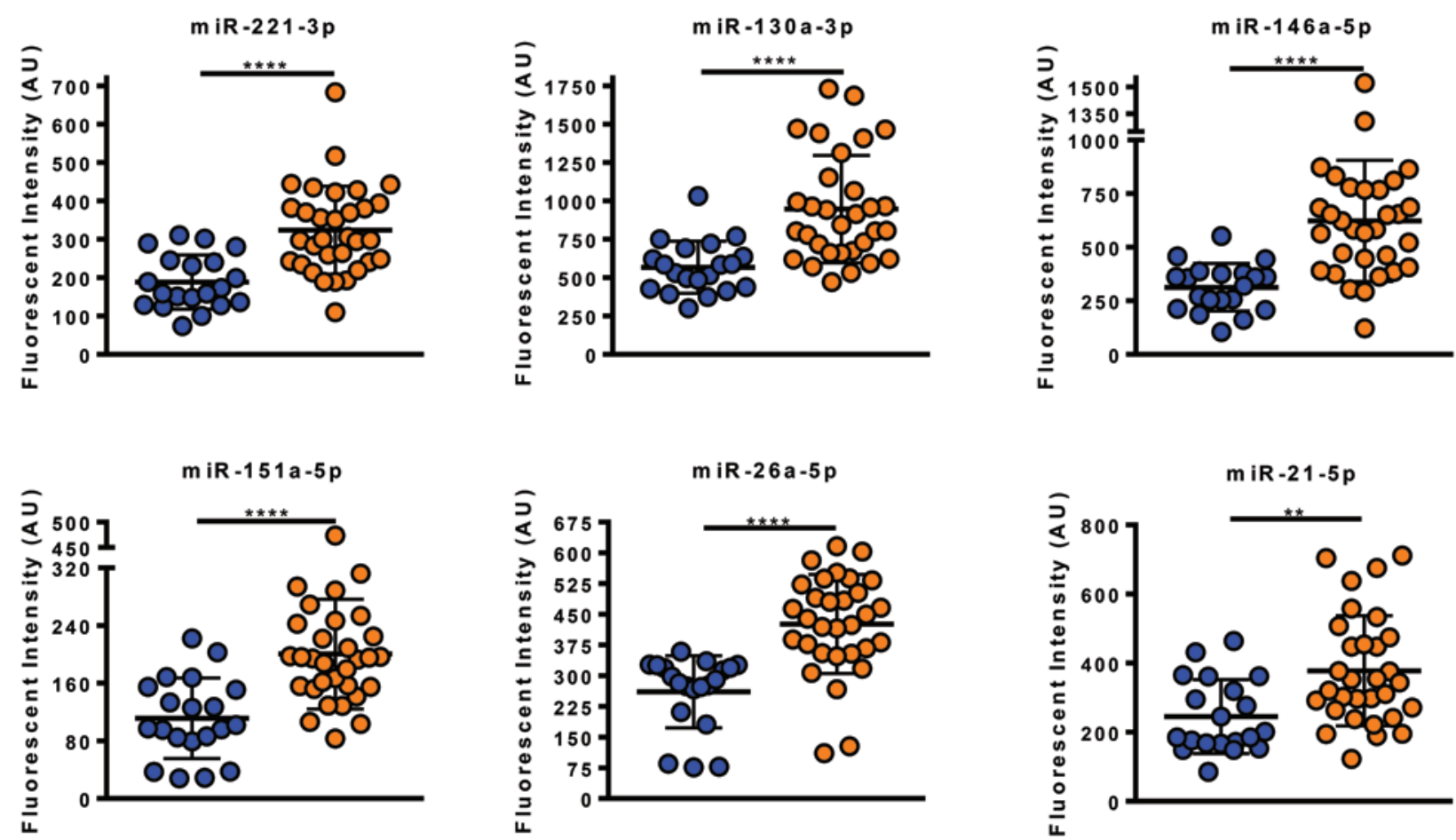

Figure 3. The expression of the 6 most differentially expressed miRNA in the serum from patients with early PsA compared to healthy controls. Relative expression of a group of 6 serum mRNA in PsA patients $(\mathrm{n}=31)$ versus HC subjects $(\mathrm{n}=20)$. $P$ values were determined using $t$ test when comparing the differences between 2 groups. ${ }^{*} P<0.05$, significantly different to comparator. AU: arbitrary units; HC: healthy controls; miRNA: microRNA; PsA: psoriatic arthritis.
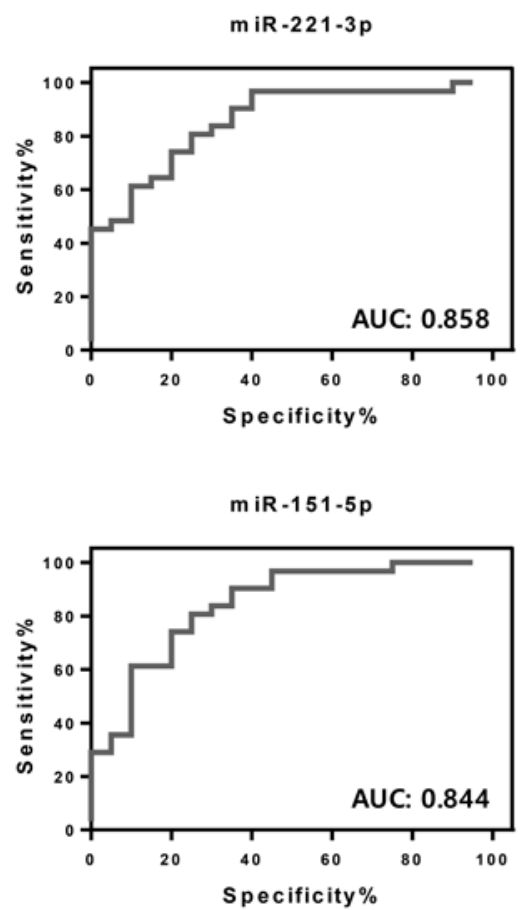
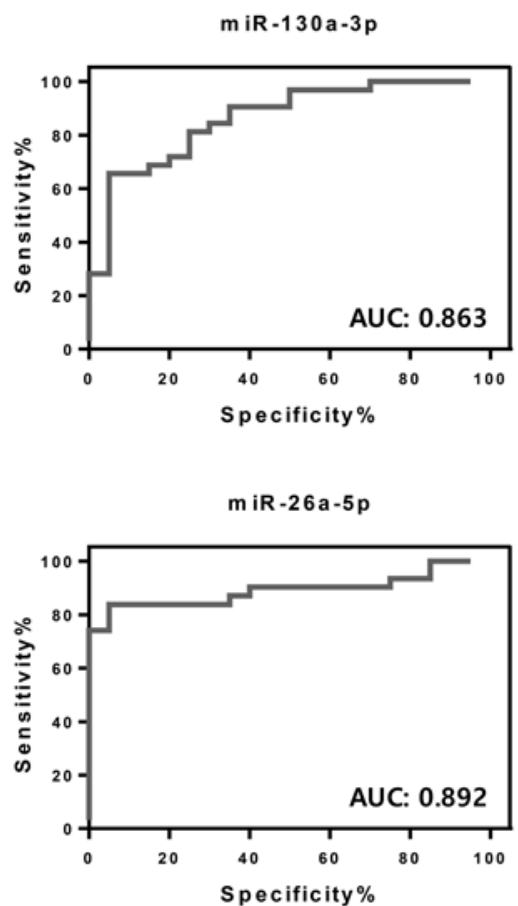

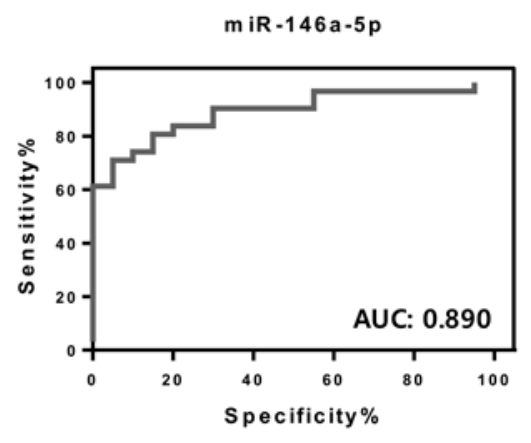

m iR -21-5p

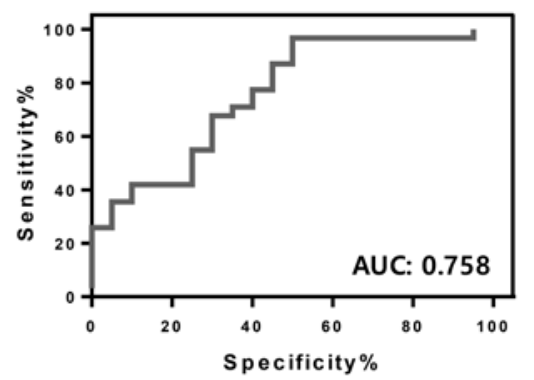

Figure 4. ROC curve analysis for PsA diagnosis. ROC curves of PsA patients $(\mathrm{n}=31)$ based on miRNA expression in serum compared to healthy control subjects $(\mathrm{n}=20)$. AUC was calculated with 95\% CI. AUC: area under the curve; PsA: psoriatic arthritis; ROC: receiver-operating characteristic. 

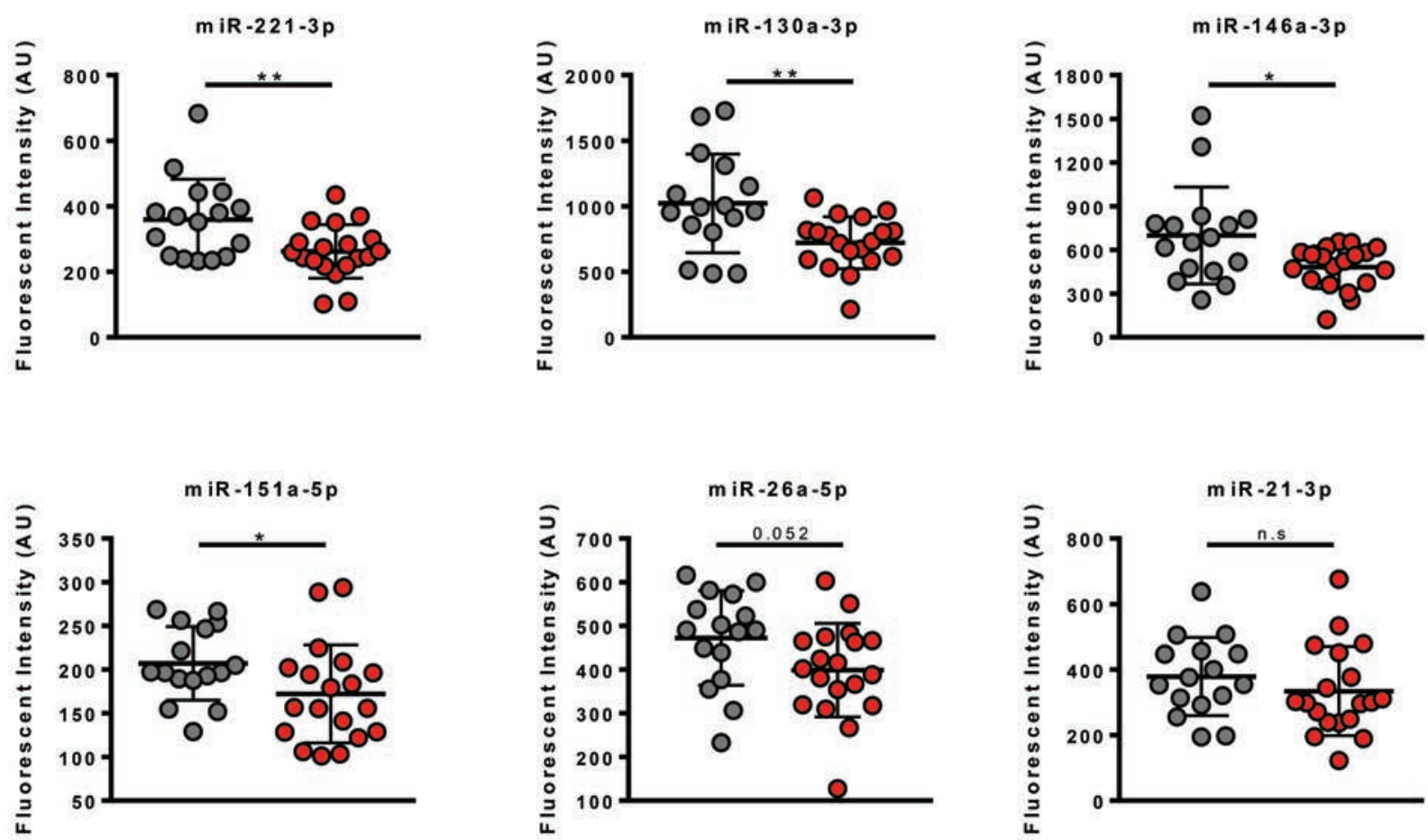

Figure 5. Relative expression of baseline serum miRNA in PsA patients with good, moderate, and nonresponse to treatment. Relative expression of a group of 6 baseline serum miRNA plotted as a function of treatment response. Nonresponding patients $(\mathrm{n}=19)$ tended to have lower baseline serum miRNA levels than in those with a good to moderate response $(\mathrm{n}=16) . P$ values were determined using $t$ test when comparing the differences between 2 groups. ${ }^{*} P<0.05$, significantly different to comparator. AU: arbitrary units; miRNA: microRNA; PsA: psoriatic arthritis; n.s.: not significant.
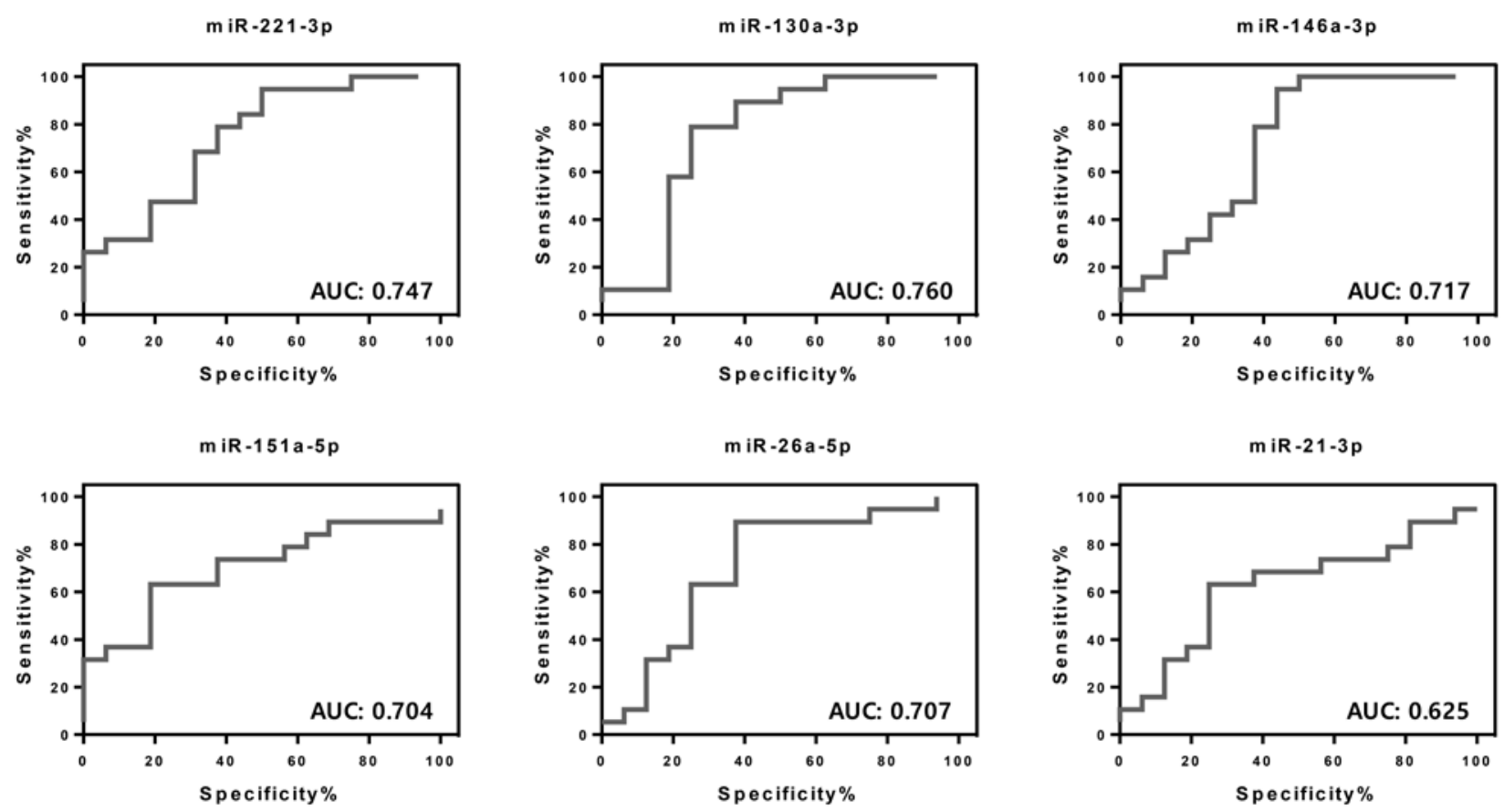

Figure 6. ROC curve analysis for baseline serum miRNA as a predictor of treatment response. AUC estimation for serum miRNA in responders $(\mathrm{n}=16)$ compared to nonresponders $(\mathrm{n}=19)$. AUC was calculated with 95\% CI. AUC: area under the curve; ROC: receiver-operating characteristic. 
miR-26a-5p (all $P<0.05$ ) discriminated PsA responders and nonresponders to treatment, with a good therapeutic response associated with a higher baseline miRNA expression (Figure 5). This discrimination was found to be independent of disease duration because miRNA levels did not correlate with disease duration. Among the patients analyzed, 49\% were receiving DMARD treatment while $51 \%$ were being treated with biologic agents. Further analysis demonstrated that any significant difference in baseline expression of miR-130a-3p, miR-221-3p, miR-146a-5p, miR-151a-5p, and miR-26a-5p and the response rates did not differ between the 2 treatment classes.

To further support these observations, ROC analysis was performed to evaluate the performance of baseline serum miRNA expression to discriminate patient treatment responses. The best performing miRNA for separation between good and moderate/nonresponder treatment outcomes were miR-221-3p, miR-130-3p, and miR-146a-3p, with AUC values of 0.747, 0.760 , and 0.717 , respectively (Figure 6). Multivariate analysis did not improve the prognostic value of this 6-miRNA signature in distinguishing patients according to treatment response (Supplementary Figure 4, available with the online version of this article).

\section{DISCUSSION}

The identification of noninvasive, convenient, and reliable biomarkers for the early diagnosis and prediction of treatment response outcomes in autoimmune disease is an area of intensive investigation. In the present study, we identified serum-derived miRNA as potential biomarkers that can support the early diagnosis of PsA patients and retrospectively stratify patients as good/moderate and nonresponders to treatment. The levels of miR-221-3p, miR-130a-3p, miR-146a-5p, miR-151-5p, miR-26a-5p, and miR-21-5p were differentially expressed in PsA patients when compared to HC subjects, with higher levels observed in patients with higher disease activity. Lower baseline levels of miR-221-3p, miR-130a-3p, miR-146a-5p, miR-151-5p, and miR-26a-5p, but not miR-21-5p, were observed in PsA with a poor therapeutic response compared to good and/or moderate responders. Among them, miR-221-3p, miR-130a-3p, and miR-146a-3p exhibited the highest accuracy in the stratification of patient response.

A number of clinical trials have previously assessed circulating miRNA as biomarkers of disease diagnosis and treatment response in a number of diseases, including cancer and type 2 diabetes $^{24,25,26}$. More recent efforts to identify serum-based biomarkers have increased as advances in technology have occurred. In addition to their stability across repeated freeze-thaw cycles $^{27}$, the ability of serum miRNA to correlate with disease activity scores are also feasible. Therefore, we hypothesized that serum miRNA could be utilized as potential biomarkers of both PsA diagnosis and treatment response. In this study, we identified for the first time to our knowledge several differentially expressed serum-derived miRNA in PsA patients compared to HC subjects. Hierarchical clustering revealed a distinguished 2-cluster miRNA heatmap profile, with most of the HC subjects clustering together in cluster 1 and PsA patients separating out into cluster 2, supporting clear miRNA expression differences between PsA and control serum. By focusing on miRNA with a significant fold change of $>1.5$ and previous associations with immunological functionalities, we identified 9 miRNA of special interest: miR-130a-3p, miR-146a-5p, miR-221-3p, miR-744-5p, miR-33a-5p, miR-151-5p, miR-26a-5p, miR-494-3p, and miR-21-5p. Interestingly, elevated levels of these miRNA were also found in early PsA patients with low disease activity, suggesting the potential value of these miRNA as biomarkers of early disease diagnosis. Many of these miRNA are also elevated in the circulation of patients with other inflammatory-driven autoimmune diseases, such as ulcerative colitis, rheumatoid arthritis (RA), and multiple sclerosis ${ }^{28,29,30}$, but have not been previously reported as a serum miRNA biomarker for PsA diagnosis. Consistent with a previous miRNA expression profile in the plasma and peripheral blood of early PsA patients ${ }^{13}$, serum-based miR-21-5p was significantly increased in early PsA patients compared to HC. Previous studies have revealed that elevated miR-21-5p is involved in psoriasis-related inflammation and modulates keratinocyte proliferation, represses $\mathrm{T}$ cell apoptosis, and promotes angiogenesis mechanisms $\mathrm{s}^{31,32,33}$. A 2014 study demonstrated that inhibition of miR-21 ameliorated dermal thickness in patient-derived psoriatic skin xenografts and psoriasis model $s^{34}$, which indicate that miR-21-5p plays a pivotal role in psoriasis development and progression. However, further experiments are required to elucidate the pathogenic mechanism of action of miR-21-5p in PsA pathogenesis. In support of the observations made in the present study, recent reports demonstrate elevated expression of miR-146a-5p in CD14+ monocytes from patients with PsA compared to both psoriasis patients and $\mathrm{HC}^{35}$, and that a single-nucleotide polymorphism in the miR-146a gene was associated with the progression of $\mathrm{PsA}^{36}$, an association that appears to be functional in patients with PsA but not $\mathrm{RA}^{37}$. Further, in terms of therapeutic response, elevated serum levels of miR-130a-3p in patients with ovarian cancer have been associated with therapeutic resistance to cisplatin ${ }^{38}$, which is consistent with other reports in glioblastoma patients and their response to a temazolamide treatment regimen ${ }^{6}$. Similarly, serum expression of miR-26a was significantly decreased in patients with psoriasis following effective treatment with the antitumor necrosis factor- $\alpha$ (TNF- $\alpha$ ) agent, etanercept ${ }^{39}$. While our findings are similar to these external validation studies, larger multicenter studies will be required to validate our findings. As the majority of PsA patients develop psoriasis first, future studies to evaluate the association between this 6-miRNA signature and psoriasis may also further understanding of their pathogenic role in disease progression.

Another novel aspect of this work is the utilization of the more clinically amenable FirePlex Biofluids miRNA assay. Previous protocols that were employed to detect circulating miRNA in patients with inflammatory arthritis relied on laborious isolation techniques, high sample volume to obtain sufficient miRNA,

Personal non-commercial use only. The Journal of Rheumatology Copyright @ 2020 . All rights reserved 
or pooled patient samples ${ }^{13,40,41,42}$. These approaches exhibit high yield variability among commercial isolation kits, require rigorous posthoc confirmation analysis, and fail to provide individual patient information ${ }^{43}$. Therefore, to date, none of these approaches have led to clinical use. In contrast to this, the FirePlex platform allows for miRNA detection in just $20 \mu \mathrm{L}$ of crude nonisolated serum that can be measured by conventional flow cytometry machines and allows for statistical analysis on individual patient miRNA levels to stratify disease groups and potentially inform clinical decisions.

Effective management in the early stages of disease will slow progression and have significant implications for improving patient quality of life, yet it remains a great challenge to identify "at-risk" or "high-priority" patients by currently available routine clinical variables. Here, we identify a set of 6 miRNA (miR-221-3p, miR-130a-3p, miR-146a-5p, miR-151-5p, miR-26a-5p, and miR-21-5p) that distinguished good/moderate and nonresponders in early PsA, independent of treatment type or duration. Interestingly, serum miRNA better stratified patients according to treatment response compared to routine variables of disease activity. In particular, our study revealed that higher baseline levels of miR-130-3p, miR-146a-3p, and miR-221-3p were associated with more optimal treatment responses. Consistent with these observations, miR-221-3p expression has been previously associated with patient prognosis and sensitivity to therapy in cancer $^{44,45}$. Moreover, miR-130a-3p was reported to modulate TNF-related, apoptosis-inducing ligand sensitivity in nonsmall cell lung cancer through MET-meditated regulation of miR-221/222 $2^{46}$. These data support the potential ability of these miRNA to improve the identification of treatment responders in early disease. However, these findings require confirmation in larger study populations. Future studies might also analyze the usefulness of serum miRNA as pharmacodynamic biomarkers to monitor response to treatment. Further, identification of the cellular source of these circulating miRNA could offer deeper insights into the functional roles of these miRNA in the pathogenesis of PsA and treatment response.

In conclusion, serum-based expression of miR-221-3p, miR-130a-3p, miR-146a-5p, miR-151-5p, miR-26a-5p, and miR-21-5p discriminated PsA patients with early disease from $\mathrm{HC}$ and successfully stratified treatment response groups from baseline levels, supporting serum miRNA as potential diagnostic and prognostic markers in PsA.

\section{ACKNOWLEDGMENT}

We are grateful to Dr. Carl Orr, Michael R. Tackett, and Francis Young for assistance with this study.

\section{ONLINE SUPPLEMENT}

Supplementary material accompanies the online version of this article.

\section{REFERENCES}

1. Pauley KM, Cha S, Chan EKL. MicroRNA in autoimmunity and autoimmune diseases. J Autoimmun 2009;32:189-94.
2. O'Connell RM, Chaudhuri AA, Rao DS, Baltimore D. Inositol phosphatase SHIP1 is a primary target of miR-155. Proc Natl Acad Sci U S A 2009;106:7113-8.

3. Taganov KD, Boldin MP, Chang K-J, Baltimore D. NFB-dependent induction of microRNA miR-146, an inhibitor targeted to signaling proteins of innate immune responses. Proc Natl Acad Sci U S A 2006;103:12481-6.

4. Wang K, Zhang S, Weber J, Baxter D, Galas DJ. Export of microRNAs and microRNA-protective protein by mammalian cells. Nucleic Acids Res 2010;38:7248-59.

5. Kleivi Sahlberg K, Bottai G, Naume B, Burwinkel B, Calin GA, Borresen-Dale A-L, et al. A serum MicroRNA signature predicts tumor relapse and survival in triple-negative breast cancer patients. Clin Cancer Res 2015;21:1207-14.

6. Chen H, Li X, Li W, Zheng H. miR-130a can predict response to temozolomide in patients with glioblastoma multiforme, independently of O6-methylguanine-DNA methyltransferase. J Transl Med 2015;13:69.

7. Gladman DD, Thavaneswaran A, Chandran V, Cook RJ. Do patients with psoriatic arthritis who present early fare better than those presenting later in the disease? Ann Rheum Dis 2011;70:2152-4.

8. Coates LC, Moverley AR, McParland L, Brown S, Navarro-Coy $\mathrm{N}$, O'Dwyer JL, et al. Effect of tight control of inflammation in early psoriatic arthritis (TICOPA): a UK multicentre, open-label, randomised controlled trial. Lancet 2015;386:2489-98.

9. Villani AP, Rouzaud M, Sevrain M, Barnetche T, Paul C, Richard $\mathrm{M}-\mathrm{A}$, et al. Prevalence of undiagnosed psoriatic arthritis among psoriasis patients: systematic review and meta-analysis. J Am Acad Dermatol 2015;73:242-8.

10. Bergman M, Lundholm A. Mitigation of disease- and treatment-related risks in patients with psoriatic arthritis. Arthritis Res Ther 2017;19:63.

11. Saber TP, Ng CT, Renard G, Lynch BM, Pontifex E, Walsh CA, et al. Remission in psoriatic arthritis: is it possible and how can it be predicted? Arthritis Res Ther 2010;12:R94.

12. Kane D, Stafford L, Bresnihan B, FitzGerald O. A prospective, clinical and radiological study of early psoriatic arthritis: an early synovitis clinic experience. Rheumatology 2003;42:1460-8.

13. Ciancio G, Ferracin M, Saccenti E, Bagnari V, Farina I, Furini F, et al Characterisation of peripheral blood mononuclear cell microRNA in early onset psoriatic arthritis. Clin Exp Rheumatol 2017; 35:113-21.

14. Wade SM, Trenkmann M, McGarry T, Canavan M, Marzaioli $\mathrm{V}$, Wade SC, et al. Altered expression of microRNA-23a in psoriatic arthritis modulates synovial fibroblast pro-inflammatory mechanisms via phosphodiesterase 4B. J Autoimmun 2019; 96:86-93.

15. Taylor W, Gladman D, Helliwell P, Marchesoni A, Mease P, Mielants H. Classification criteria for psoriatic arthritis: development of new criteria from a large international study. Arthritis Rheum 2006;54:2665-73.

16. Fransen J, van Riel PL. The Disease Activity Score and the EULAR Response Criteria. Rheum Dis Clin North Am 2009;35:745-57.

17. Glintborg B, Østergaard M, Krogh NS, Andersen MD, Tarp U, Loft AG, et al. Clinical response, drug survival, and predictors thereof among 548 patients with psoriatic arthritis who switched tumor necrosis factor $\alpha$ inhibitor therapy: results from the Danish Nationwide DANBIO Registry. Arthritis Rheum 2013;65:1213-23.

18. Ademowo OS, Hernandez B, Collins E, Rooney C, Fearon U, van Kuijk AW, et al. Discovery and confirmation of a protein biomarker panel with potential to predict response to biological therapy in 
psoriatic arthritis. Ann Rheum Dis 2016;75:234-41.

19. Collins ES, Butt AQ, Gibson DS, Dunn MJ, Fearon U, van Kuijk AW, et al. A clinically based protein discovery strategy to identify potential biomarkers of response to anti-TNF- $\alpha$ treatment of psoriatic arthritis. Proteomics Clin Appl 2016;10:645-62.

20. Ng CT, Biniecka M, Kennedy A, McCormick J, FitzGerald O, Bresnihan B, et al. Synovial tissue hypoxia and inflammation in vivo. Ann Rheum Dis 2010;69:1389-95.

21. Chapin SC, Appleyard DC, Pregibon DC, Doyle PS. Rapid microRNA profiling on encoded gel microparticles. Angew Chem Int Ed Engl 2011;50:2289-93.

22. Pritchard CC, Kroh E, Wood B, Arroyo JD, Dougherty KJ, Miyaji MM, et al. Blood cell origin of circulating microRNAs: a cautionary note for cancer biomarker studies. Cancer Prev Res 2012;5:492-7.

23. Vandesompele J, De Preter K, Pattyn F, Poppe B, Van Roy N, De Paepe A, et al. Accurate normalization of real-time quantitative RT-PCR data by geometric averaging of multiple internal control genes. Genome Biol 2002;3:RESEARCH0034.

24. Bagnoli M, Canevari S, Califano D, Losito S, Maio MD, Raspagliesi F, et al. Development and validation of a microRNA-based signature (MiROvaR) to predict early relapse or progression of epithelial ovarian cancer: a cohort study. Lancet Oncol 2016;17:1137-46.

25. Montani F, Marzi MJ, Dezi F, Dama E, Carletti RM, Bonizzi G, et al. miR-Test: a blood test for lung cancer early detection. J Natl Cancer Inst 2015;107: djv063.

26. Zhu H, Leung SW. Identification of microRNA biomarkers in type 2 diabetes: a meta-analysis of controlled profiling studies. Diabetologia 2015;58:900-11.

27. Balzano F, Deiana M, Dei Giudici S, Oggiano A, Baralla A, Pasella $S$, et al. miRNA stability in frozen plasma samples. Molecules 2015;20:19030-40.

28. Wu F, Guo NJ, Tian H, Marohn M, Gearhart S, Bayless TM, et al. Peripheral blood microRNAs distinguish active ulcerative colitis and Crohn's disease. Inflamm Bowel Dis 2011;17:241-50.

29. Stanczyk J, Pedrioli DML, Brentano F, Sanchez-Pernaute O, Kolling C, Gay RE, et al. Altered expression of microRNA in synovial fibroblasts and synovial tissue in rheumatoid arthritis. Arthritis Rheum 2008;58:1001-9.

30. Gandhi R, Healy B, Gholipour T, Egorova S, Musallam A, Hussain MS, et al. Circulating microRNAs as biomarkers for disease staging in multiple sclerosis. Ann Neurol 2013;73:729-40.

31. Ichihara A, Jinnin M, Yamane K, Fujisawa A, Sakai K, Masuguchi $S$, et al. microRNA-mediated keratinocyte hyperproliferation in psoriasis vulgaris. Br J Dermatol 2011;165:1003-10.

32. Liu LZ, Li C, Chen Q, Jing Y, Carpenter R, Jiang Y, et al. MiR-21 induced angiogenesis through AKT and ERK activation and HIF-1 $\alpha$ expression. PLoS One 2011;6:e19139.

33. Meisgen F, Xu N, Wei T, Janson PC, Obad S, Broom O, et al. MiR-21 is up-regulated in psoriasis and suppresses $\mathrm{T}$ cell apoptosis. Exp Dermatol 2012;21:312-4.
34. Guinea-Viniegra J, Jiménez M, Schonthaler HB, Navarro R, Delgado Y, Concha-Garzón MJ, et al. Targeting miR-21 to treat psoriasis. Sci Transl Med 2014;6: 225re1.

35. Lin S-H, Ho J-C, Li S-C, Chen J-F, Hsiao C-C, Lee C-H. MiR-146a-5p expression in peripheral CD14+ monocytes from patients with psoriatic arthritis induces osteoclast activation, bone resorption, and correlates with clinical response. J Clin Med 2019;8:110.

36. Maharaj AB, Naidoo P, Ghazi T, Abdul NS, Dhani S, Docrat TF, et al. MiR-146a G/C rs2910164 variation in South African Indian and Caucasian patients with psoriatic arthritis. BMC Med Genet 2018;19:48.

37. Xiao Y, Liu H, Chen L, Wang Y, Yao X, Jiang X. Association of microRNAs genes polymorphisms with arthritis: a systematic review and meta-analysis. Biosci Rep 2019;39:BSR20190298.

38. Chen C, Wang H, Yang L-Y, Jia X, Xu P, Chen J, et al. [Expression of MiR-130a in serum samples of patients with epithelial ovarian cancer and its association with platinum resistance]. [Article in Chinese] Sichuan Da Xue Xue Bao Yi Xue Ban 2016;47:60-3.

39. Pivarcsi A, Meisgen F, Xu N, Ståhle M, Sonkoly E. Changes in the level of serum microRNAs in patients with psoriasis after antitumour necrosis factor- $\alpha$ therapy. Br J Dermatol 2013; 169:563-70.

40. Castro-Villegas C, Pérez-Sánchez C, Escudero A, Filipescu I, Verdu M, Ruiz-Limón P, et al. Circulating miRNAs as potential biomarkers of therapy effectiveness in rheumatoid arthritis patients treated with anti-TNF $\alpha$. Arthritis Res Ther 2015;17:49.

41. Hruskova V, Jandova R, Vernerova L, Mann H, Pecha O, Prajzlerova $\mathrm{K}$, et al. MicroRNA-125b: association with disease activity and the treatment response of patients with early rheumatoid arthritis. Arthritis Res Ther 2016;18:124.

42. Murata K, Yoshitomi H, Tanida S, Ishikawa M, Nishitani K, Ito H, et al. Plasma and synovial fluid microRNAs as potential biomarkers of rheumatoid arthritis and osteoarthritis. Arthritis Res Ther 2010;12:R86.

43. Moldovan L, Batte KE, Trgovcich J, Wisler J, Marsh CB, Piper M. Methodological challenges in utilizing miRNAs as circulating biomarkers. J Cell Mol Med 2014;18:371-90.

44. Khella HWZ, Butz H, Ding Q, Rotondo F, Evans KR, Kupchak P, et al. miR-221/222 Are Involved in Response to Sunitinib Treatment in Metastatic Renal Cell Carcinoma. Mol Ther 2015;23:1748-58.

45. Garofalo M, Quintavalle C, Romano G, Croce CM, Condorelli G. miR221/222 in cancer: their role in tumor progression and response to therapy. Curr Mol Med 2012;12:27-33.

46. Acunzo M, Visone R, Romano G, Veronese A, Lovat F, Palmieri $\mathrm{D}$, et al. miR-130a targets MET and induces TRAIL-sensitivity in NSCLC by downregulating miR-221 and 222. Oncogene 2012;31:634-42. 